\title{
Analysis and Simulation on the Kinematics of Robot Dexterous Hand
}

\author{
Jin Guo ${ }^{1}$, Ni Jiangnan ${ }^{1}$ \\ ${ }^{1}$ Dept. of mechanical and electrical automation, Henan Polytechnic Institute, Nanyang Henan, 473000, China;
}

Key words: robot dexterous hand, kinematics, analysis, simulation, MATLAB

\begin{abstract}
Dexterity" is an important index to measure the technical level of the robot dexterous hand, which demonstrates the ability to work in any position with the target shape. This study is based on the Shadow dexterous hand, and the mechanical structure characteristics of Shadow dexterous hand are studied. Then the kinematics model is established by using $\mathrm{D}-\mathrm{H}$ parameter method, and the forward and inverse kinematics equations are derived. Finally the results are verified and simulated in MATLAB.
\end{abstract}

\section{Introduction}

At present, the massage devices on the market are to play a role in relaxation and fatigue relief, but they have little effect on the treatment of disease. Therefore, it has a broad market prospect to develop a robot dexterous hand with professional level of traditional Chinese medicine massage by using robot technology [1-3]. The Chinese medicine massage robot dexterous hand has to be provided with enough dexterity to reach the practical level. With the development of robot technology, the research of dexterous hand has made great progress and the Shadow dexterous hand produced by British Shadow Company is one of the typical representatives [4-6].

In this paper, the structural characteristics of the Shadow dexterous hand were analyzed, and the kinematic model was established. The forward and inverse kinematics of dexterous hand were studied and the analytic solutions were solved. The results are validated and simulated by using MATLAB software to provide theoretical support for the application of dexterous hand technology in traditional Chinese medicine massage.

\section{Shadow Dexterous Hand}

The Shadow dexterous hand used in this study, as shown in Figure 1 and 2, consists of 24 joints and a total of 20 degrees of freedom. It is driven by 40 pneumatic-muscles-actuators(PMA) and transited by tendons and integrates joint position sensors, pressure sensors and fingertip tactile array sensors.

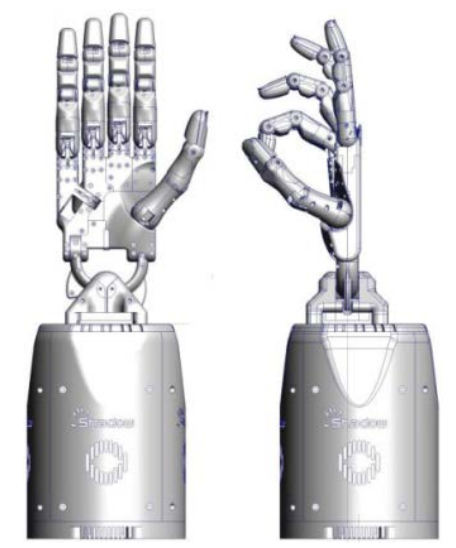

Fig. 1 the appearance of Shadow dexterous hand 
The wrist of Shadow dexterous hand has 2 joints and 2 degrees of freedom. The thumb has 5 joints and 5 degrees of freedom. Each of the other fingers has 4 joints and 3 degrees of freedom, and the little finger has an extra joint in the palm provided to allow opposition to the thumb.

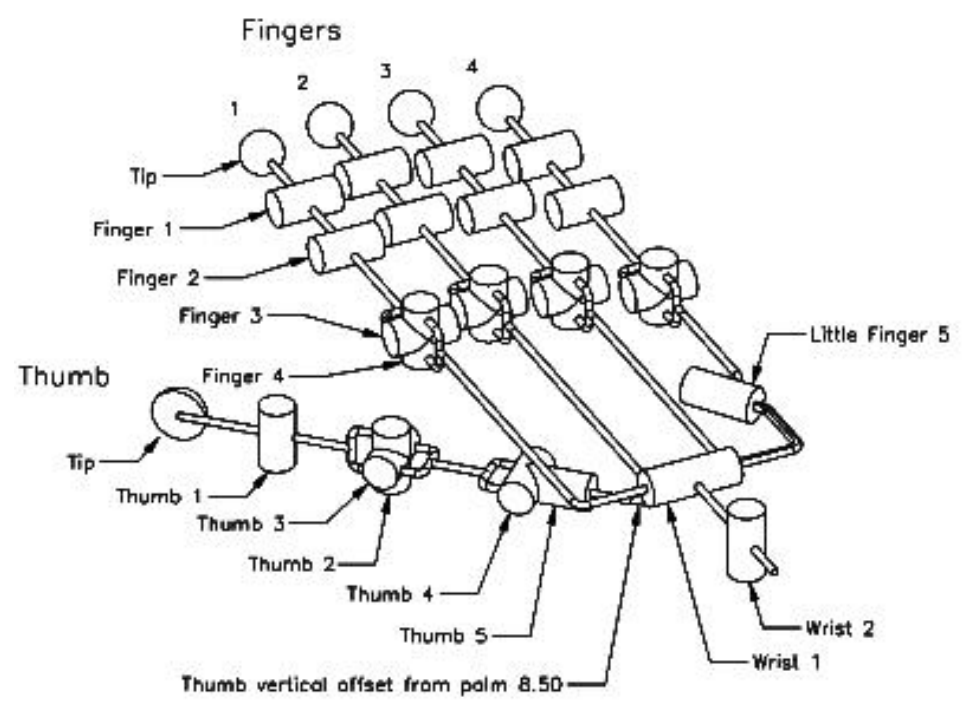

Fig. 2 the structure of Shadow dexterous hand

\section{Kinematic Analysis of the Finger}

In order to control the motion trajectory of the robot dexterous hand, the analysis of kinematics parameters is needed, so that the quantitative relation between the position of the fingertip and the angle of each joint can be established [7-8]. In the following sections, the middle finger is taken as an example to introduce the establishment of coordinate system and the research of forward and inverse kinematic parameters, and the validation and simulation of the kinematic of middle finger are also finished. The research process of the other finger is similar to middle finger, so they are not repeated here [9-10].

The coordinate system of the dexterous hand. The middle finger is taken as an example, and the movement of the finger is completed by the joints and the connecting rods. The middle finger coordinate system is shown in Figure 3 , and the coordinate system $O_{0}-x_{0} y_{0} z_{0}$ and $O_{t}-x_{t} y_{t} z_{t}$ are base coordinate system and fingertip coordinate system respectively.

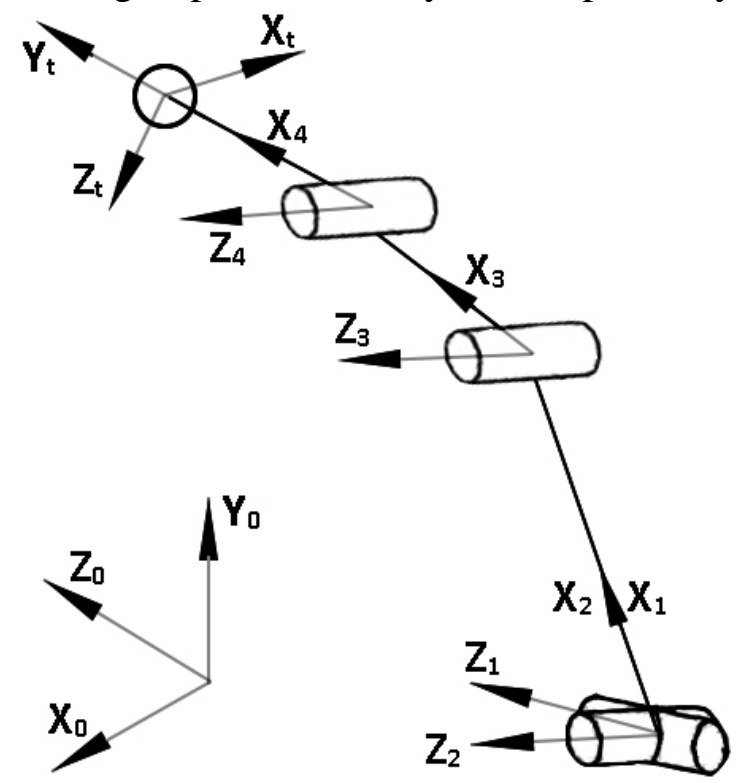

Fig. 3 Middle-finger link coordinates of Shadow dexterous hand 
The forward kinematics of middle finger. The $\mathrm{D}-\mathrm{H}$ parameters of middle finger is shown in Table 1.

Table 1. D-H parameters of middle-finger

\begin{tabular}{|c|c|c|c|c|}
\hline joint & $d_{i}$ & $a_{i-1}$ & $\alpha_{i-1}$ & $\theta_{i}$ \\
\hline 1 & 0 & 0 & 0 & $\theta_{1}$ \\
\hline 2 & 0 & 0 & $90^{\circ}$ & $\theta_{2}$ \\
\hline 3 & 0 & $a_{2}$ & 0 & $\theta_{3}$ \\
\hline 4 & 0 & $a_{3}$ & 0 & $\theta_{4}$ \\
\hline
\end{tabular}

According to the parameters in Table 1, the transformation matrices between the adjacent link-pole coordinate can be obtained, and the position transition matrix between the coordinate system $O_{0}-x_{0} y_{0} z_{0}$ and $O_{t}-x_{t} y_{t} z_{t}$ is described in Equation 1.

$$
\begin{aligned}
& { }_{t}^{0} T={ }_{1}^{0} T_{2}^{1} T_{3}^{2} T_{4}^{3} T_{t}^{4} T=\left[\begin{array}{cccc}
n_{x} & o_{x} & a_{x} & x_{t} \\
n_{y} & o_{y} & a_{y} & y_{t} \\
n_{z} & o_{z} & a_{z} & z_{t} \\
0 & 0 & 0 & 1
\end{array}\right] \\
& =\left[\begin{array}{cccc}
s_{1} & c_{1} c_{234} & -c_{1} s_{234} & a_{4} c_{1} c_{234}+a_{3} c_{1} c_{23}+a_{2} c_{1} c_{2} \\
-c_{1} & s_{1} c_{234} & -s_{1} s_{234} & a_{4} s_{1} c_{234}+a_{3} s_{1} c_{23}+a_{2} s_{1} c_{2} \\
0 & s_{234} & c_{234} & a_{4} s_{234}+a_{3} s_{23}+a_{2} s_{2} \\
0 & 0 & 0 & 1
\end{array}\right] .
\end{aligned}
$$

In the equation, $s_{i}, c_{i}, s_{i j}, c_{i j}, s_{i j k}$ and $c_{i j k}$ represent $\sin \theta_{i}, \cos \theta_{i}, \sin \left(\theta_{i}+\theta_{j}\right)$, $\cos \left(\theta_{i}+\theta_{j}\right), \sin \left(\theta_{i}+\theta_{j}+\theta_{k}\right)$, and $\cos \left(\theta_{i}+\theta_{j}+\theta_{k}\right)$ respectively $(\mathrm{i}, \mathrm{j}, \mathrm{k}=1,2,3,4)$. The movement range of each joint's angular displacement is here: $-25^{\circ} \leq \theta_{1} \leq 25^{\circ},-10^{\circ} \leq \theta_{2} \leq 90^{\circ}$, $0^{\circ} \leq \theta_{3} \leq 90^{\circ}, 0^{\circ} \leq \theta_{4} \leq 90^{\circ}$.

The inverse kinematics of middle finger. The inverse kinematics is based on the posture and position of the finger to deduce the numerical value of each joint's angular displacement [11].

Supposed the position of fingertip in base coordinate system is $\left(x_{t}, y_{t}, z_{t}\right)$, Equation 2 and 3 can be deduced from Equation 1.

$$
\begin{aligned}
& \theta_{1}=\arctan \frac{y_{t}}{x_{t}} . \\
& \frac{x_{t}}{c_{1}}=\frac{y_{t}}{s_{1}}=a_{4} c_{234}+a_{3} c_{23}+a_{2} c_{2} .
\end{aligned}
$$

Equation 4 and 5 can be deduced from Equation 1 and 3.

$$
\begin{aligned}
& \theta_{2}=\operatorname{atan} 2\left(z_{t}, \pm \sqrt{\left(a_{4} s_{34}+a_{3} s_{3}\right)^{2}+\left(a_{3} c_{34}+a_{3} c_{3}+a_{2}\right)^{2}-z_{t}^{2}}\right)-\operatorname{atan2}\left(a_{4} s_{34}+\right. \\
& \left.a_{3} s_{3}, a_{3} c_{34}+a_{3} c_{3}+a_{2}\right) . \\
& \frac{c_{4}}{a_{2}}+\frac{c_{34}}{a_{3}}+\frac{c_{3}}{a_{4}}=\frac{\left(\frac{x_{t}}{c_{1}}\right)^{2}+z_{t}{ }^{2}-a_{2}{ }^{2}-a_{3}{ }^{2}-a_{4}{ }^{2}}{2 a_{2} a_{3} a_{4}} .
\end{aligned}
$$


Due to $\theta_{3}=\theta_{4}$, Equation 5 should be substituted by Equation 6, which can be used to get $\cos \theta_{3}$ and $\theta_{3}$.

$$
\frac{2 c_{3}^{2}}{a_{3}}+\frac{\left(a_{2}+a_{4}\right) c_{3}}{a_{2} a_{4}}=\frac{\left(\frac{x_{t}}{c_{1}}\right)^{2}+z_{t}^{2}-a_{2}^{2}-a_{3}^{2}-a_{4}^{2}+2 a_{2} a_{4}}{2 a_{2} a_{3} a_{4}} .
$$

\section{Verification and Simulation of Kinematics}

The MATLAB software is used to establish three-dimensional simulation mode and verify and test the kinematics research results [12].

Kinematics verification. The functions link and robot are used to establish the simulation mode of middle finger, and the functions fkine is used to obtain the absolute value of forward kinematics and verify kinematics functions.

Two sets of selected parameters are used to verify the kinematics equations - Set $\mathrm{A}$ is $\theta_{1}=\frac{\pi}{2}$, $\theta_{2}=\frac{\pi}{2}, \theta_{3}=\frac{\pi}{2}$, and $\theta_{4}=\frac{\pi}{2}$, and Set $\mathrm{B}$ is $\theta_{1}=\frac{23 \pi}{36}, \theta_{2}=\frac{\pi}{4}, \theta_{3}=\frac{\pi}{6}$, and $\theta_{4}=\frac{\pi}{6}$. The validation results are shown in Table 2 with the numerical values of $\theta_{1}, \theta_{2}, \theta_{3}$, and $\theta_{4}$ mentioned above. $\mathrm{M}$ indicates the results from MATLAB, and $C$ shows the calculation results from Equation 1.

Table 2. Validation results of middle-finger forward kinematics

\begin{tabular}{|c|c|c|c|c|c|c|c|}
\hline Set & & $x_{t} / \mathrm{mm}$ & $y_{t} / \mathrm{mm}$ & $z_{t} / \mathrm{mm}$ & $a_{x}$ & $a_{y}$ & $a_{z}$ \\
\hline \multirow{2}{*}{$\mathrm{A}$} & $\mathrm{M}$ & 0 & -25 & 20 & 0 & 1 & 0 \\
\cline { 2 - 8 } & $\mathrm{C}$ & 0 & -25 & 20 & 0 & 1 & 0 \\
\hline \multirow{2}{*}{$\mathrm{B}$} & $\mathrm{M}$ & -13.4476 & 28.8385 & 80.1161 & 0.4082 & -0.8754 & -0.2588 \\
\cline { 2 - 8 } & $\mathrm{C}$ & -13.45 & 28.84 & 80.12 & 0.41 & -0.88 & -0.26 \\
\hline
\end{tabular}

Compared with the two groups of data, the following conclusions can be reached: the calculation results agree well with the theoretical values and the forward kinematic model and equation of middle finger are reliable.

Kinematics simulation. The functions plot and drivebot are used to draw the three-dimensional mode of middle finger and simulate the kinematics. Figure 4 is the simulated images corresponding to the two sets of validation data in Table 2.

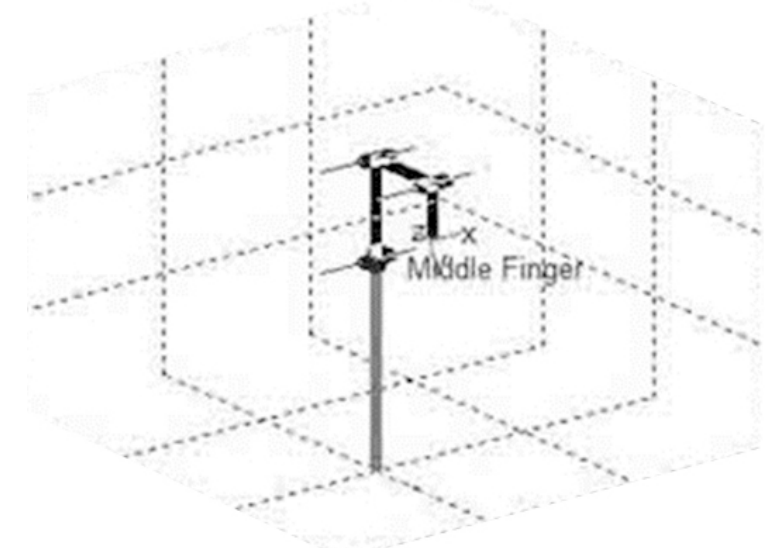

(1)

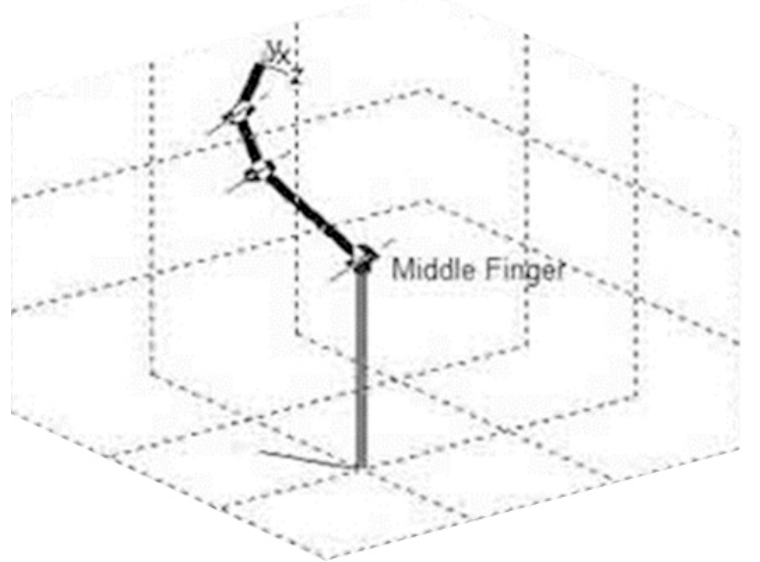

(2)

Fig. 4 Simulation of forward kinematics 


\section{Summary}

The shadow dexterous hand is taken as the research object, and the kinematics model of middle finger is established by using the D-H coordinate method. The forward and inverse kinematics equations are deduced and the research results are verified and simulated in MATLAB software. The research conclusion shows that the Shadow dexterous hand is able to satisfy the functional requirement of Chinese medicine massage robot hand and the data in this paper can provide reference for the research of the massage robot.

\section{Reference}

[1] J.G. Luo, M.Y. He, Z.X. Xue, et al. Current situation and challenges on the study of dexterous hand. Journal of Machine Design, 2014(10): 4-8.

[2] Z. Yang, Y.S. Hua. Research on the control of a new type dexterous hand with two fingers. Science and technology innovation herald, 2013(18): 13-14.

[3] R.T. Xing, Z.J. Sun, C.S. Zhao, et al. Motion Planning of the Fingers of a Five-fingered Dexterous Hand with Independent Joints. Mechanical Science and Technology, 2013, 27(3): 370-373.

[4] Y.L. Zhu, B.J. Guo. Kinematics calculation and analysis of multi-fingered dexterous hand driven by artificial muscles. Machinery Design and Manufacture, 2013(11): 224-227.

[5] H. Wang. Kinematics analysis and test of underwater dexterous hand. Journal of Mechanical Transmission, 2012, 36(3): 67-69.

[6] L. Jiang, H. Liu. Inverse kinematics of dexterous finger with linkage mechanism. Journal of Harbin Institute of Technology, 2014, 39(3): 394-397.

[7] J.T. Li, Y.R. Zhang. Motion mapping in master-slave grasp for dexterous hands. Journal of Beijing University of Aeronautics and Astronautics, 2015, 31(7): 722-725.

[8] J. Liu, H.X. Sun, Y.R. Zhang. Mapping human hand motion to dexterous robotic hands. Journal of Beijing University of Posts and Telecommunications, 2015, 28(2): 54-58.

[9] J.J. Luo, G.Q. Hu. Study on the simulation of robot motion based on MATLAB. Journal of Xiamen University (Natural Science), 2015, 44(5): 640-644.

[10] Y. Xue, Z. Lu. The design and simulation analysis of a new dexterous hand. Dual Use Technologies and Products, 2014(4): 44-46.

[11] C. Qin, W.Z. Yang, H. Zhang, et al. interactive motion analysis of the ywz dexterous hand. Journal of Zhejiang Sci-tech University, 2012, 29(6): 832-836.

[12] D.H. Gao, X.B. Hu. The Analysis of Kinematic Simulation of 6-DOF Articulated Carrier Robot Based on ADAMS. Manufacture Information Engineering of China, 2013, 39(1): 37-39. 